\title{
Mass Deposition after Pellet Injection into a Tokamak
}

\author{
V. Rozhansky ${ }^{1}$ I. Senichenkov ${ }^{1}$, I. Veselova $^{1}$, R. Schneider $^{2}$ \\ ${ }^{1}$ St. Petersburg State Polytechnical University, St. Petersburg, 195251, Russia \\ ${ }^{2}$ Max-Planck Institut für Plasmaphysik, Teilinstitut Greifswald, EURATOM Association, \\ D-17491 Greifswald, Germany
}

\section{Introduction}

Pellet injection is considered to be a prospective way to provide refueling of future tokamaks and stellarators. The density profile, which is created after fast processes of mass averaging of the injected plasma over the flux surfaces, affects both the fusion power and the confinement regime. As was observed in several experiments on tokamaks the ablated material after fast mass averaging processes is shifted significantly with respect to the flux surfaces, where the pellet ablation took place. The observed shift is reported to be in the low field side (LFS) direction and the main cause of the shift is supposed to be $\nabla B$ acceleration of the ablated material. This acceleration is caused by cloud polarization increasing with time and space in the inhomogeneous magnetic field and predicted to be [1,2] $g=2 c_{s}^{2} / R$, where $R$ is a tokamak major radius and $c_{s}=\sqrt{\left(T_{e}+T_{I}\right) / m_{I}}$ is a sound speed.

However, there is experimental evidence that the shift in the LFS direction is inconsistent with the constant acceleration of the ablatant. Two mechanisms to reduce cloud polarization are considered: so-called Alfven conductivity of the ambient plasma $[1,3]$ and the current inside the cloud driven by anomalous viscosity.

As demonstrated below the mass deposition after the pellet injection is mainly determined by the plasma acceleration in the LFS direction, Alfven conductivity of the background plasma and compensation of the $\nabla B$ currents in different parts of the cloud propagating along the magnetic field. The analytical expression for the shift is derived and the numerical simulations in the simplified model are performed.

\section{Simplified physical model}

Let's consider a pellet cloud motion in a reference frame of a moving pellet (the corresponding condition was discussed in [1]) and assume the cloud to have constant electron and ion temperatures, which are much less than the ambient one. Since the ablated particles drift velocity $V_{R}$ towards LFS is significantly higher than pellet velocity $V_{p}$, the pellet may be considered as almost a constant source slowly changing its intensity in time. The current balance equation for the vertical currents (see Fig. 1) may be written as 


$$
\frac{m_{I} N}{B^{3}} E_{y} \frac{d E_{y}}{d x}=-2 \Sigma_{A}\left(E_{y}-E_{0}\right)+\frac{2 N\left(T_{e}+T_{I}\right)}{B R}
$$

where $N=\int_{-\infty}^{+\infty} n \mathrm{~d} z$ is the density integrated along magnetic field lines, $E_{y}$ is the vertical electric field due to polarization of the cloud. The 1.h.s. is the polarization current inside the cloud (the term with time derivative is neglected since the plasma flow should be stationary near the constant particle source). The last term in the r.h.s. is the vertical $\nabla B$ current. The first term in the r.h.s. is the polarization current of the Alfven wave in the ambient plasma, spreading along the magnetic field lines. $\Sigma_{A}=\sqrt{m_{i} n / \mu_{0}} / B$. It is caused by so-called Alfven conductivity, which determines the ability of the ambient plasma to reduce polarization of the cloud, $n_{i}$ and $m_{i}$ are the density and the ion mass of the ambient plasma, and $E_{0}=V_{p} B$.

Far from the pellet, where the electric field becomes large enough and the $\nabla B$ current may be balanced by the polarization current in the Alfven wave, the ablated particles move in the LFS direction with the constant velocity (in a laboratory reference frame)

$$
V_{R}^{(\infty)}=\frac{N\left(T_{e}+T_{I}\right)}{\Sigma_{A} B^{2} R}
$$

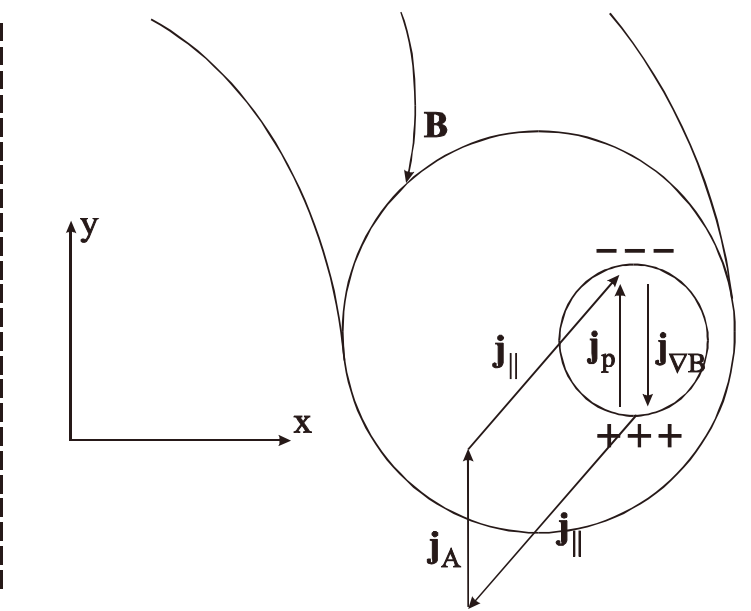

Figure 1. Polarization of the ablatant and the polarization currents of the Alfven wave in the ambient plasma.

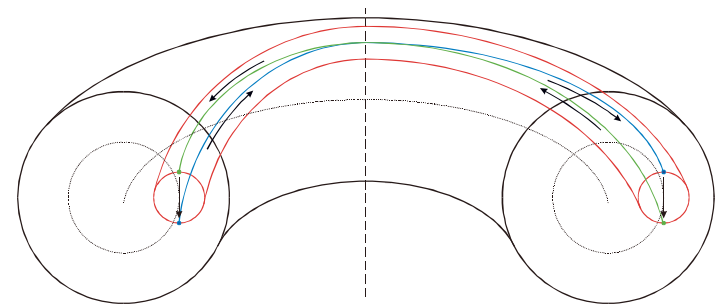

Blue and green lines represent two magnetic field lines. Black arrows demonstrate compensation of the $\operatorname{grad} B$ current inside the cloud.

Figure 2. Scheme of the cloud spreading along the magnetic field lines.

The density of the ablatant $N$ integrated along magnetic field lines is connected with the pellet ablation rate $N$ by the particle continuity equation. Consider the neutral cloud surrounding a pellet as a source of the ionized particles $P=N \exp \left(-\left(x^{2}+y^{2}+z^{2}\right) / l_{i}^{2}\right) / \pi^{3 / 2} l_{i}^{3}$. Then the particle continuity equation integrated along $z$ and $x$ at $y=0$ is $N V_{R}=\dot{N} / \sqrt{\pi} l_{i}$. Combining this with (2), we find 


$$
V_{R}=V_{R}^{(\infty)}=c_{s} \sqrt{\frac{\dot{N} m_{I}}{B R l_{i}} \sqrt{\frac{\mu_{0}}{\pi n_{i} m_{i}}}} .
$$

After the poloidal turn at the angle $\pi$ due to expansion along $\vec{B}$ two magnetic field lines that originally were at the top and bottom of the cloud will change their positions to the opposite ones (Fig. 2). Hence the net perpendicular current flowing from the first to the second field line due to $\nabla B$ is reduced with respect to the second term in the r.h.s. of Eq. (1). The velocity $V_{R}$ should also decrease at a time scale $t=R / c_{s}$. The motion in the $x$ direction is cancelled at this moment and the final mass displacement may be estimated as

$$
\Delta x=V_{R}^{(\infty)} R / c_{s}=\sqrt{\frac{m_{I} \dot{N}}{B}} \cdot \sqrt[4]{\frac{\mu_{0} R c_{s}}{4 \pi D n_{i} m_{i}}} .
$$

We also replaced here the ionization radius $l_{i}$ by the diffusive length $l_{D}=\sqrt{4 D R / c_{s}}$.

\section{Results of numerical simulation}

To test the role of the effects considered the 2D system for the ionized ablatant density integrated along the magnetic field lines and for the polarization potential $\Psi(x, y)$ was simulated (see also [1]).

$$
\begin{aligned}
& \frac{\partial N}{\partial t}+(\nabla \cdot N \vec{u})=Q(x, y)+D \Delta N \\
& \left(\nabla \cdot\left(\frac{\vec{B} \times\left(T_{e}+T_{I}\right) \nabla N}{B^{2}}-\frac{m_{i} N}{B^{2}} \frac{\mathrm{d} \nabla \Psi}{\mathrm{d} t}-\frac{m_{i}}{B^{2}}(\nabla \Psi \cdot Q)+\vec{I}_{A}+\vec{I}_{v i s}\right)\right)=0
\end{aligned}
$$

Here $Q=\int_{-\infty}^{+\infty} P(x, y, z) \mathrm{d} z, \frac{\mathrm{d}}{\mathrm{dt}}=\frac{\partial}{\partial t}+(\vec{u} \cdot \nabla), \vec{u}=\frac{\vec{B} \times \nabla \Psi}{B^{2}}, I_{A} \overrightarrow{\mathrm{y}}=-2 \Sigma_{A} \frac{\partial \Psi}{\partial y} \overrightarrow{\mathrm{y}}, B=B_{0}\left(1-\frac{2 x}{R}\right)$. The anomalous diffusion is taken into account by the last term in the r.h.s. of Eq. (5). In the expression for viscous current $\vec{I}_{v i s c}=\vec{B} \times\left(\nabla \cdot \pi^{(A N)}\right) / B^{2}$ the Braginski [5] perpendicular viscosity is used with the classical coefficient replaced by the anomalous value.

The results of the simulation are presented in Fig. 3, for three combinations of parameters: 1) $D=0, n_{i}=0$; 2) $D=1 \mathrm{~m}^{2} / \mathrm{s}, n_{i}=0$; 3) $D=1 \mathrm{~m}^{2} / \mathrm{s}, n_{i}=10^{20} \mathrm{~m}^{-3}$ The other parameters were: $B=2.5 T, R=2 \mathrm{~m}, l_{i}=1 \mathrm{~cm}, c_{s}=10^{4} \mathrm{~m} / \mathrm{s}, \dot{N} \approx 1.37 \cdot 10^{24} \mathrm{~s}^{-1}$. One can see (Fig. 3a) that the viscosity and diffusivity don't change significantly the value of the acceleration. However with account of Alfven conductivity after a certain time the acceleration of the cloud becomes zero and cloud moves with a constant velocity (Fig. 3b), which is close to that given by Eq. (3). 
The shift of the ablated material measured on DIII-D and ASDEX-Upgrade is reported to be about $25-30 \mathrm{~cm}$ for both the cases. The same shift gives Eq. (4) for the parameters of the experiments. For the ITER parameters (assuming a spherical pellet with a radius $5 \mathrm{~mm}$ and injection velocity $300 \mathrm{~m} / \mathrm{s}$ ) the penetration depth is about $20 \mathrm{~cm}$, while the displacement of the ablated material towards the LFS given by (4) is $\Delta x \approx 2.1 \mathrm{~m}$.
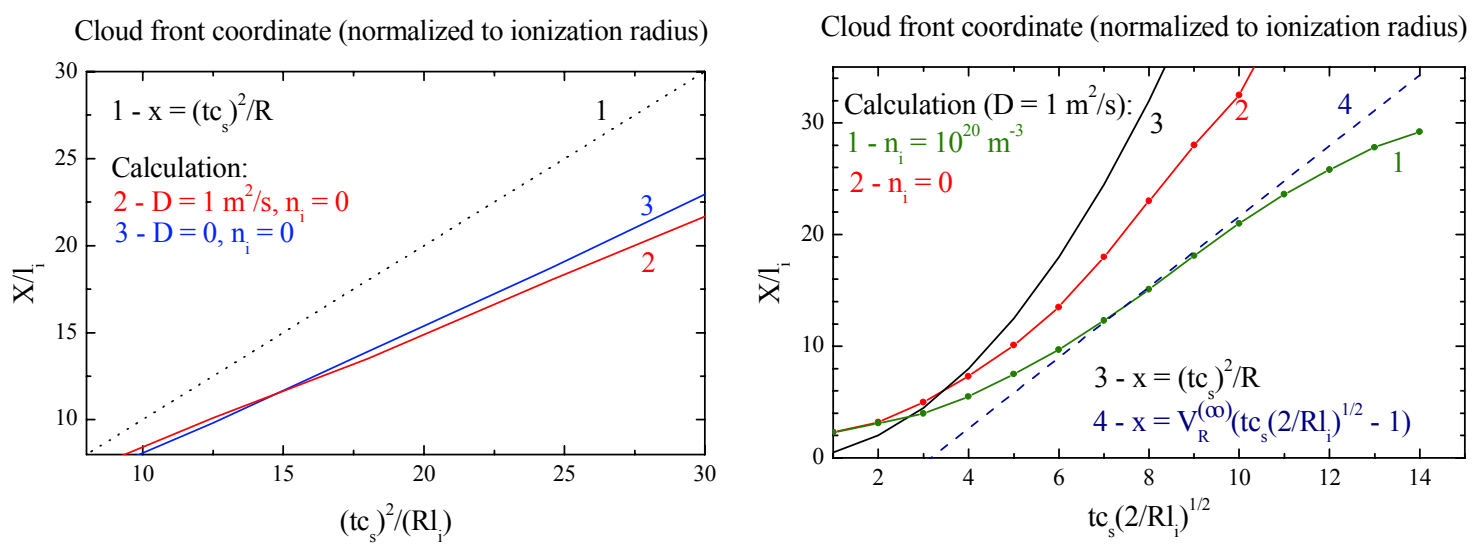

Figure 3. Cloud front coordinate: a) as a function of $t^{2}$; b) as a function of $t$.

It is worthwhile mentioning that Eq. (4) gives only an expression for the shift of the front of the plasma jet towards the LFS. In reality the material is deposited in between the position of this front and the ablation point. To predict the density profile after the pellet injection one needs to consider the cloud expansion along the magnetic field lines with account of the shape of magnetic flux surfaces. The corresponding code is under development.

\section{Acknowledgements}

The work was performed in the framework of Russian-German cooperation (WTZ project number RUS574) and was supported by Russian Foundation for Basic Research (Grant 0102-16651 and 03-02-06741) and Russian Ministry of Education (Grant E02-3.2-300). The authors are grateful to Dr L. Lengyel for fruitful discussions.

\section{References}

[1] V.Rozhansky et al., Plasma Phys. Control. Fusion 37, 399 (1995).

[2] L.L. Lengyel, Nucl. Fusion 17, 805 (1977).

[3] P.B. Parks et al., Phys. Plasmas 7, 1968 (2000).

[4] M. Scholer, Planet. Space Sci. 18, 977 (1980).

[5] S. I. Braginskii, in Rev. Plasma Phys. Ed. by M.A. Leontovich, Consultants Bureau N.Y. v. 1. 1965, p.205. 